\title{
On the Postulational Basis of the Theory of Elementary Particles*
}

\author{
H. J. ВнавнA \\ Tata Institute of Fundamental Research, Bombay, India
}

\begin{abstract}
A set of postulates is formulated which leads to the relativistic wave equations of present quantum mechanics. The mathematical difference between the usual non-linear interaction terms and terms which introduce essential non-linearities in the equations is discussed. It is proved that every particle must possess an antiparticle unless at least one of the basic postulates is discarded. The connection between the rest mass of the particle, the minimal equation of the $\alpha$-matrices, and the general commutation relations of the $\alpha$ matrices is derived and discussed. It is proved that for a particle of spin $\frac{1}{2}$ there is only one possible wave equation, while for a particle of spin 1 there are others besides the usual scalar and vector equations. One such example is given.
\end{abstract}

\section{INTRODUCTION}

$\mathrm{E}^{\mathrm{x}}$ XPERIENCE has shown that the attempt to derive the laws of nature by pure thought and epistemological reasoning has neither met with much success, nor proved particularly fruitful in stimulating a growth of our knowledge of nature. On the other hand, as a result of the development of physics since the time of Newton, it has come to be recognized that the varied and multifold results of observation can only be unified into a coherent and related scheme by laying down as the laws of nature certain mathematical statements which may not be capable of direct verification, but the deductions from which can be compared with experiment. The aim of theoretical physics must therefore be to find a complete set of mutually consistent mathematical postulates or axioms from which the properties of nature, meaning thereby the result of every conceivable experiment, can be deduced in the form of a series of theorems. It is, however, necessary in order to achieve the last step of comparing the mathematical statements of the theorems with the results of observation, that the basic mathematical postulates must be supplemented by a set of prescriptions about the interpretation of the mathematical formalism. It is clearly not sufficient that the postulates should be consistent, and their correctness from the point of view of physics can only be demonstrated by an agreement between the deductions and the results of experiment.

Present relativistic quantum theory has evolved out of a generalization of the classical Hamilton-Jacobi formulation of Newtonian mechanics to include the indeterminacy principle on the one hand, and the principle of special relativity on the other. It is well known that the resulting theory is not a completely consistent scheme, since it leads to inconsistencies such as an infinite self-energy for every elementary particle. It has nevertheless had remarkable success in describing a very wide range of phenomena in nature, and in pre-

* This paper is based on a report prepared by the author for the Solvay Conference, 1948. It contains certain new results besides many well-known theorems which are proved here to be of wider validity than might have been thought. dicting the existence of the positron and of the processes of pair creation and annihilation. It is therefore of interest, notwithstanding the self-energy difficulties, to inquire what relativistic wave equations can be set up within the frame work of the existing scheme. Such an investigation is necessary inasmuch as it may provide a description, within the limits of the present theory, of some of the new elementary particles that are now being discovered. It would indeed be remarkable if these new particles were all described by the Dirac equation, or in addition by the two known relativistic equations for particles of spin 0 and 1 , and if this should turn out to be true, one would have to find some explanation of why other and more complicated wave equations for the elementary particles do not play a role in the description of nature. The aim of this paper is to attempt to formulate, necessarily briefly, the postulates which appear to underly the present scheme of relativistic quantum theory and to summarize the different work on relativistic wave equations for the elementary particles which has been done within the framework of these postulates.

It is clearly impossible within the scope of a short paper to adhere strictly to a program of the type described in the first paragraph, and one is compelled, even at the expense of a certain apparent loss in logical rigor, to state the fundamental postulates in words which already carry an implication about the interpretation of the formalism. The justification for this procedure is not only that it touches what is of greatest interest to the physicist, but rather that it goes directly to the root of the matter, and it is not difficult to see how the postulates given below can be replaced by a set of purely mathematical definitions and postulates on the one hand, and a set of prescriptions identifying the mathematical symbols or expressions with physical quantities on the other.

\section{THE BASIC POSTULATES AND GENERAL THEORY}

One may take as the first postulate underlying the theory, the demand of relativistic invariance: 
Postulate 1. The predictions of the theory with regard to the result of any possible observation shall be in accordance with the requirements of the principle of special relativity.

This postulate does not by itself require that the properties of Cartesian space-time shall hold in regions of space which are of nuclear or sub-nuclear dimensions. It only expresses the fact that all observations, even of atomic phenomena, are ultimately made with the help of macroscopic measuring instruments, and for these there is considerable experimental evidence that the principle of special relativity must hold. In practice, however, one usually takes postulate 1 in the more restrictive form ${ }^{1}$ of Postulate $1^{\prime}$. The mathematical formalism shall be covariant throughout for all transformations of the orthochronous ${ }^{2}$ Lorents group.

The full Lorentz group $\mathscr{L}$ is the set of all real transformations which leaves invariant the real quadratic form

$$
\left(x^{0}\right)^{2}-\left(x^{1}\right)^{2}-\left(x^{2}\right)^{2}-\left(x^{3}\right)^{2},
$$

and consists of four disconnected pieces. If this expression is equal to $a^{2}>0$, then either $x^{0}>|a|$ or $x^{0}<-|a|$ and the transformations of the full Lorentz group must therefore fall into two disconnected pieces, one consisting of all transformations which preserve the sign of $x^{0}$, which we name the orthochronous transformations, and the other of all those which reverse the sign of $x^{0}$, called here the antichronous transformations. It is easy to see that the former piece forms a sub-group $\mathcal{L} \uparrow$ of the full Lorentz group which it is convenient to call the orthochronous Lorentz group. A different division of the group into two parts is obtained as a result of the well-known fact that the determinant of any transformation of the full Lorentz group is equal to \pm 1 , so that all those transformations whose determinant is +1 and those whose determinant is -1 belong to two disconnected pieces. The former forms a sub-group known as the proper Lorentz group $\mathfrak{L}_{+}$. This splitting into two pieces according to the sign of the determinant can be applied to each of the two pieces consisting of the orthochronous and antichronous transformations only. We thus get four disconnected pieces in all, of which one consisting of the set of all transformations of determinant +1 which preserve the sign of the time component $x^{0}$ of a time like vector forms a sub-group by itself. This sub-group we call the orthochronous proper Lorentz group $\mathfrak{L}_{+} \uparrow$.

Since a change from one system to another in which the direction of the time axis is reversed does not appear to be possible in nature, it is sufficient and necessary to demand that the formalism shall be covariant for the orthochronous Lorentz group $\mathscr{2}^{\uparrow}$ only.

The second postulate concerns the existence of a wave function from which all properties of the physical system can be deduced. It may be formulated as

Postulate 2. A function $\psi$, called the wave function, whose value is defined at all points of a space-like surface, exists such that it provides the maximum possible amount of information which can be obtained by observation about the state of the physical system.

In this connection it should be noted that the wave function may have several components $\psi_{1}, \psi_{2}, \cdots \psi_{\nu}$, each component being a function defined at all points

\footnotetext{
1 This presupposes that certain obvious definitions of spacetime consistent with postulate 1 have been made.

${ }^{2}$ From the Greek words ópós, upright, and $\chi \rho o$ ovos, time.
}

of the same space-like surface. In this case $\psi$ is to be regarded as a matrix of one column and $\nu$-rows, the element in the $\sigma$ th row being $\psi_{\sigma}$. In postulating that the values of the wave function can be given at all points of a space-like surface, we may be assuming more than can possibly be determined by observation, and later developments may show that it is necessary to abandon this postulate. However, it is difficult to reject the postulate as definitely incorrect at this stage, since our general approach only requires that the deductions from the basic postulates should be in agreement with observation. The wave function itself may simply be a mathematical tool with the help of which the fundamental laws can be formulated.

The aim of predicting the future state of a system from its present state then leads directly to:

Postulate 3. The fundamental laws of nature are such that given the values of the wave function $\psi$ at all points of a space-like surface, it is possible to calculate its values at all points of a later space-like surface.

It should be noted that this postulate is somewhat wider than the usual one implying the existence of a differential equation in space-time. However, for the present, we replace postulate 3 by a more limited one which embodies in it the idea of near action, namely, that the change with time in the value of the wave function at any point of a space-like surface shall depend only on its values in the immediate neighborhood. In mathematical language, we replace postulate $3 \mathrm{by}^{3}$

Postulate $3^{\prime}$. The fundamental equations of physics are partial differential equations which allow one to calculate the values of the wave function on a later space-like surface when they are given on an earlier space-like surface.

It then follows from the first two postulates that the equations cannot contain higher derivatives of the wave function than the first. For if they contained higher derivatives of the wave function with respect to the time than the first, then it would not be possible to calculate the wave function on a later space-like surface unless at least its first-order time-like derivative on the earlier surface were given. But this would imply the ability to determine more about the state of the system on the original surface than is contained in the wave function, contrary to the second postulate. The first postulate next requires that no derivatives of the wave function with respect to the space-coordinates of order higher than the first should occur in the wave equations, since space and time have to be on the same footing. Later experience may require us to abandon postulate $3^{\prime}$ for the more general postulate 3 .

The result of every observation is by its very nature a real quantity, whereas no such requirement has been made of the wave-function $\psi$. In order to obtain an expression which is always real from a complex quantity, one has to make use of its complex conjugate $\bar{\psi}$.

${ }^{3}$ This postulate again presupposes that the wave function is continuous and differentiable throughout space-time, except perhaps on a finite number of points, lines, or surfaces. 
One should therefore expect $\psi$ as well as its complex conjugate $\bar{\psi}$ to play a part in the formulation of the basic laws.

One must now formulate some statement embodying the observation that space-time, at least for all but galactic distances, is homogeneous and isotropic. By this we mean that all points of space-time are equivalent except insofar as this equivalence is destroyed by the presence of physical entities. Likewise, one must postulate that all directions in space-time which can be transformed into each other in accordance with postulate $1^{\prime}$ are equivalent. In mathematical language this becomes

Postulate 4. Besides the wave function $\psi$, its complex conjugate, and their derivatives, no functions varying with the coordinates and no constants dependent on the choice of the frame of reference shall enter in the equations of motion. Equivalently, the equations of motion shall only contain universal constants besides the wave function $\psi$, its complex conjugate and their derivatives.

Next we take as

Postulate 5. The equations of motion can be derived by the variation of a Lagrangian which is the integral over a four-dimensional space-time volume of a real Lagrange function, which is itself a function of the components of the wave function $\psi$, their complex conjugates, and their derivatives with respect to the coordinates.

In obtaining the variation of the Lagrangian a certain nuance has to be observed. We consider every component $\psi_{\sigma}$ of $\psi$ to be defined by the relation $\psi_{\sigma}=u_{\sigma}+i v_{\sigma}$. where $u_{\sigma}$ and $v_{\sigma}$ are two independent real functions of the coordinates. Similarly, we define $\bar{\psi}_{\sigma}=u_{\sigma}-i v_{\sigma}$. One then calculates the variation of the Lagrangian for arbitrary and independent variations of $u_{\sigma}$ and $v_{\sigma}$ at each point, and equates this to zero to obtain the equations of motion. It is easy to see that this procedure leads to the same equations as one would obtain by varying $\psi_{\sigma}$ and $\bar{\psi}_{\sigma}$ as if they were independent functions. It then follows from these equations that $u_{\sigma}$ and $v_{\sigma}$ can be treated as the real and imaginary parts of one analytic function $\psi_{\sigma}$. This is the rigorous formulation of the usual procedure adopted by physicists of varying $\psi$ and $\bar{\psi}$ in the Lagrangian as if they were unconnected functions.

We next make a postulate which appears to be necessary if we are to obtain the present scheme of quantum mechanics and not something more general. Moreover, it provides the foundation upon which the usual methods of perturbation theory in quantum mechanics can be based.

Postulate 6. The Lagrange function is a rational integral function of the components of the wave function $\psi$, $\bar{\psi}$ and their derivatives with respect to the coordinates.

This postulate requires that the Lagrange function be a sum of terms, each of which is a product of positive integral powers of the components of $\psi, \bar{\psi}$, and their derivatives. Each such term may be multiplied by some universal constant. We note in this connection that derivatives of $\psi$ or $\bar{\psi}$ of higher order than the first cannot appear in the Lagrange function since otherwise the equations obtained by a variation of the Lagrangian would be of higher order than the first, contrary to postulate 2. Similarly, while a term can be of any integral degree 4 in $\psi$, it must be only of zero or first degree in the derivatives of $\psi$ since otherwise the equations would again be of order higher than the first, contrary to postulate 2 .

Since there is only one Lagrangian from which the equations of motion have to be deduced, it follows from postulate $1^{\prime}$ that this Lagrangian must be an invariant, and in consequence, every term of the Lagrange function of which it is an integral must itself be an invariant. We therefore start to build the Lagrange function by first finding all possible terms of first degree in $\psi$ which are invariant, then all the possible invariant terms of second degree, then of the third degree, and so on.

Let $Q$ and $Q^{\prime}$ be two Lorentz frames of reference related to each other by the corresponding relation between the coordinates $x^{k}$ and $x^{\prime k}$, respectively, of the same point,

$$
x^{k}=t_{l}^{k} x^{l}
$$

where a Latin index stands for $0,1,2$, or 3 , and $t_{l}^{k}$ are the elements of a matrix of the Lorentz group defined by

$$
t_{r}^{k} t_{s} g^{r s}=g^{k l},
$$

the metric tensor being assumed to have the form $g_{00}=-g_{11}=-g_{22}=-g_{33}=1, g_{k l}=0$ for $k \neq l .{ }^{5}$ Then, according to postulate $1^{\prime}$ the values, in the two systems, of the wave functions $\psi$ and $\psi^{\prime}$ at the same point must be connected by a linear relation of the type

$$
\psi^{\prime}=T \psi
$$

where $T$ is a $\nu \times \nu$-matrix. As is well known, for every transformation $t_{l}{ }^{k}$ in (1) there is a corresponding matrix $T$ in (2), and the set of matrices $T$ forms a representation $R$ of the Lorentz group. An irreducible representation of the orthochronous Lorentz group is characterized by two numbers $n$ and $m$ such that $n \geqslant m \geqslant 0$, and both are integers or both half-odd integers. For integral $n, m$ the representation gives the transformations of a tensor of rank $n+m$ that is, having $n+m$ tensor indices (or $2 n$ dotted plus undotted spinor indices) and maximum symmetry properties, while for half-odd integral $n, m$, it corresponds to a spinor of rank $2 n$, (that is one having $2 n$ dotted plus undotted spinor indices) and maximum symmetry properties. Denote this representation by $R(n, m)$. The representation $R$ is either one of these or it is reducible, in which case by a

4 "The degree $\delta$ in $\psi$ " of a term is, as usual, defined as: If in this term every component of $\psi, \bar{\psi}, \partial \psi / \partial x^{k}$, and $\partial \bar{\psi} / \partial x^{k}$ is replaced by $\lambda$ times itself, $\lambda$ being any real number, then the term is changed to $\lambda^{\delta}$ times its original value. Similarly, "the degree of a term in the derivatives of $\psi$ " is defined as the number $\delta$ in the factor $\lambda^{\delta}$ by which the term is multiplied if every component of $\partial \psi / \partial x^{k}$ and $\partial \bar{\psi} / \partial x^{k}$ is replaced by $\lambda$ times itself, the components of the wave functions $\psi$ and $\bar{\psi}$ being unchanged.

${ }^{5}$ Summation from 0 to 3 is understood over every repeated Latin index appearing both above and below in the same formula, but not over repeated indices appearing only above or only below. 
well-known theorem it must be fully reducible and expressible as a direct sum of such irreducible representations :

$$
R=R(n, m)+R\left(n^{\prime}, m^{\prime}\right)+\cdots
$$

We take $\Omega$ to be already in its fully reduced form (3).

Suppose now that $R$ contains the representation $R(0,0)$, that is, that one of the components of $\psi$, say $\psi_{\sigma}$ is an invariant. Then this component of $\psi$ remains unchanged by any transformation $T$ and in consequence the matrix $\Gamma$ of one row and $\nu$-column having 1 in the $\sigma$ th column and zeros everywhere else has the property that $\Gamma \psi$ is an invariant. It follows that even when $\psi$ is not in its fully reduced form, a matrix $\Gamma$ of one row and $\nu$-columns must exist such that $\Gamma \psi$ is an invariant, $\Gamma$ having the same invariant matrix form in all systems (though not the form just given). If the representation $\Re(0,0)$ appears $\mu$-times in $R$, then there are $\mu$-independent matrices $\Gamma_{(1)}, \Gamma_{(2)}, \cdots \Gamma_{(\mu)}$ such that $\Gamma_{(1)} \psi$, $\Gamma_{(2)} \psi, \cdots \Gamma_{(\mu)} \psi$, are all independent invariants.

Next suppose that $\mathcal{R}_{k}$ contains the representation $R(1,0)$, that is, four components transforming equivalently to a four-vector. Then, by a similar reasoning, it can be proved that four matrices $\Gamma^{k}$ of one row and $\nu$-columns and having the same matrix form in all systems exist such that the four quantities $\Gamma^{k} \psi$ transform like a four-vector. Similar statements are true for every irreducible representation in (3). For example, if $R$ contains the representation $R(1,1)$, then six matrices $\Gamma^{k l}=-\Gamma^{l k}$ of one row and $\nu$-columns exist such that $\Gamma^{k} \psi$ form the six components of a skew-symmetric tensor of second rank.

It is convenient at this stage to introduce the notation $\partial_{k}=\partial / \partial x^{k}, p_{k}=-i \partial_{k}$, where the factor $i$ has simply been introduced for convenience. We adopt the convention of understanding $\mathbf{p}_{k}$ to mean $i \partial_{k}$ operating on any function to the left of $\mathbf{p}_{k}$.

Returning to the Lagrange function, we note that the only terms of first degree which can be contained in it are of type $c \Gamma \psi+\overline{c \Gamma \psi}, c$ being some arbitrary universal constant. But the variation of such terms only lead to the addition of a constant in certain of the equations of motion, and by themselves lead to nothing interesting. Linear terms of the type $\Gamma^{k} \partial_{k} \psi$ in the Lagrange function can be ignored, since being perfect differentials their integrals can be transformed immediately into surface integrals and contribute nothing to the equations of motion.

We construct next the quadratic terms in $\psi$ in the Lagrange function. These can be divided into three classes, the first class consisting of all terms of the type $\bar{\psi}_{\sigma} \psi_{\rho}$, or $\bar{\psi}_{\sigma} p_{k} \psi_{\rho}$, or $\bar{\psi}_{\sigma} \mathrm{p}_{k} \psi_{\rho}$, the second class of all terms of the type $\psi_{\sigma} \psi_{\rho}$ or $\psi_{\sigma} p_{k} \psi_{\rho}$, and the third class of all terms of the type $\bar{\psi}_{\sigma} \bar{\psi}_{\rho}$ or $\bar{\psi}_{c} \mathbf{p}_{k} \bar{\psi}_{\rho}$.

First consider only terms of the first class. Any sum of terms of the type $\bar{\psi}_{\sigma} \psi_{\rho}$ can always be written in the form $\psi^{\dagger} \gamma \psi$ where $\gamma$ is a $\nu \times \nu$-matrix. ${ }^{6}$ Since the Lagrange

\footnotetext{
${ }^{6}$ A dagger affixed to a matrix denotes its Hermitian conjugate, thus $\psi^{\dagger}$. A curl on top of a matrix denotes its transposed, thus $\tilde{\psi}$. A bar over a matrix denotes its complex conjugate.
}

function must be real, $\psi^{\dagger} \gamma \psi$ is either always real, in which case $\gamma$ is Hermitian, or if not, we must add its complex conjugate $\overline{\psi^{\dagger} \gamma \psi}$ to the function. But $\overline{\psi^{\dagger} \gamma \psi}$ $=\psi^{\dagger} \gamma^{\dagger} \psi$ so that in effect we have in the Lagrange function $\psi^{\dagger}\left(\gamma+\gamma^{\dagger}\right) \psi$. We then simply write $\gamma$ in place of the Hermitian matrix $\gamma+\gamma^{\dagger}$. Thus, the matrix $\gamma$ must be Hermitian. Moreover, in accordance with postulate $1^{\prime}$ this expression must be an invariant for all transformations of the type (2). Hence, the matrix $\gamma$ must satisfy

$$
T^{\dagger} \gamma T=\gamma
$$

for every transformation of $R$. Next consider terms of the type $\bar{\psi}_{\sigma} p_{k} \psi_{\rho}$. All such terms can be written in the form $\psi^{\dagger} \gamma^{k} p_{k} \psi$, where the $\gamma^{k}$ are four $\nu \times \nu$-matrices. These matrices must be Hermitian again. For

$$
\psi^{\dagger} \gamma^{k} p_{k} \psi=p_{k}\left(\psi^{\dagger} \gamma^{k} \psi\right)+\psi^{\dagger} \mathbf{p}_{k} \gamma^{k} \psi
$$

In order to ensure that the. Lagrange function is real we must add the complex conjugate, namely,

$$
-p_{k}\left(\overline{\psi^{\dagger} \gamma^{k} \psi}\right) \overline{\psi^{\dagger} \mathrm{p}_{k} \gamma^{k} \psi}
$$

But $\overline{\psi^{\dagger} \mathbf{p}_{k} \gamma^{k} \psi}=\psi^{\dagger} \gamma^{k \dagger} p_{k} \psi$. Thus, in effect one again has $\psi^{\dagger}\left(\gamma^{k}+\gamma^{k \dagger}\right) p_{k} \psi$ in the Lagrange function. The $\gamma^{k}$ are therefore necessarily Hermitian matrices again. In this case, $\psi^{\dagger} \gamma^{k} \psi$ is a real number and therefore the first terms in (5a) and (5b) cancel each other. They would in any case contribute nothing to the equations of motion as they are perfect differentials. Moreover, terms of the type $\psi^{\dagger} \gamma^{k} p_{k} \psi$ and $\psi^{\dagger} \mathbf{p}_{k} \gamma^{k} \psi$ are equivalent as far as the variation principle is concerned. Thus, all terms of the first class can be written in the form

$$
\psi^{\dagger} \gamma^{k} p_{k} \psi+\psi^{\dagger} \gamma \psi
$$

where $\gamma$ and the four $\gamma^{k}$ are Hermitian matrices. It can be shown now that all quadratic terms in $\psi$, including those of the second and third class, can be written in the form (6).

Consider the quadratic terms of the type $\psi_{\sigma} p_{k} \psi_{\rho}$ in the Lagrange function. All such terms can be written in the form $\tilde{\psi} \eta^{k} p_{k} \psi$, where it can be shown by an argument similar to that used above that $\eta^{k}$ must be skew-symmetric: $\eta^{k}=-\eta^{k}$. To this term must be added its conjugate complex $\overline{\widetilde{\psi} \eta^{k} p_{k} \psi}$. Writing $\psi=u+i v$ where $u$ and $v$ are two real column matrices of $\nu$-rows each, and introducing a new one-column matrix $\phi=u \dot{+} v$ with $2 \nu$-rows it is easy to see that

where

$$
\begin{gathered}
\tilde{\psi} \eta^{k} p_{k} \psi+\overline{\widetilde{\psi} \eta^{k} p_{k} \psi}=\phi^{\dagger} \xi^{k} p_{k} \phi \\
\zeta^{k}=\left(\begin{array}{cc}
\eta^{k}+\eta^{k \dagger} & i\left(\eta^{k}-\eta^{k \dagger}\right) \\
i\left(\eta^{k}-\eta^{k \dagger}\right) & -\left(\eta^{k}+\eta^{k \dagger}\right)
\end{array}\right)
\end{gathered}
$$

is a $2 \nu \times 2 \nu$ Hermitian matrix. It follows that all terms of the type considered can be written in the form of the first term in (6) where now the components of the matrix $\phi$ are all real instead of complex. All such terms can therefore be included in an expression of the type (6) where some of the components of $\psi$ are purely real, and it therefore constitutes a particular case of the more general expression (6). The same holds for all terms of the type $\tilde{\psi} \eta \psi+\bar{\psi} \eta \psi$ which can be written in the form $\phi^{\dagger} \zeta \phi$. 
Postulate $1^{\prime}$ regarding relativistic invariance now requires that under any transformation of the type (1) and (2) the $\gamma^{k}$ should transform as:

$$
T^{\dagger} \gamma^{k} T=t_{l}^{k} \gamma^{l} \text {. }
$$

Just as in the case of the $\Gamma$ 's, there may be several linearly independent matrices $\gamma_{(1)}, \gamma_{(2)}, \cdots$ satisfying (4), and therefore as many linearly independent terms of the type $\psi^{\dagger} \gamma_{(\mu)} \psi$ multiplied by arbitrary universal constants can be introduced in the Lagrange function. The $\gamma$ in (6) is to be understood as a sum of all these, the universal constants being absorbed therein. A similar remark applies to the $\gamma^{k}$ in (6).

The $\gamma$ and $\gamma^{k}$ in (6) are arbitrary to the extent of equivalence. If $a$ be any non-singular $\nu \times \nu$-matrix, then the similarity transformation

$$
\psi^{\prime}=a \psi
$$

transforms the $\gamma^{\prime}$ 's to the equivalent forms

$$
\gamma^{\prime}=a^{\dagger} \gamma a, \quad \gamma^{\prime k}=a^{\dagger} \gamma^{k} a
$$

and in consequence of (4) and (7) the matrices of the representation $R$ are transformed to the equivalent form

$$
T^{\prime}=a^{-1} T a .
$$

The matrices $\gamma, \gamma^{k}$ may form an irreducible set under transformations of the type (8). In that case the only arbitrariness that still remains, but for equivalence transformations of the type (8), is that $\gamma$ may be multiplied by an arbitrary real factor $\chi$. It is convenient to choose such a factor to have the same dimensions as $p_{k}$, that is, of a reciprocal length, so that the matrices $\gamma$ and $\gamma^{k}$ may be treated as of the same dimensions, or rather, as dimensionless quantities. An arbitrary real factor $c$ mulitplying the four $\gamma^{k}$ would be trivial since it could be removed by replacing $\psi$ by $\sqrt{|c|} \psi$, and this would simply result in a change of the factor multiplying $\gamma$. The $\gamma^{k}$ cannot be multiplied by different factors since they are connected by relations of the type (7).

If the set of matrices $\gamma^{k}$ and $\gamma$ is reducible, then it must be fully reducible under transformations of the type (8), that is, expressible as a direct sum of irreducible sets. This simply follows from the fact that the $\gamma$ 's are Hermitian matrices. In that case (6) breaks up into a sum of a number of expressions of the same type, each involving a separate set $\psi_{(1)}$, $\psi_{(2)}, \cdots$ of the components of $\psi$. However, such a decomposition is not of significance unless the representation $R$ is likewise decomposed in a corresponding way, for otherwise it would not be relativistically invariant. Thus, the physically significant reduction is when the matrices $\gamma$ as well as the representation $R$ decompose into a direct sum of irreducible sets, corresponding to the existence of a number of independent types of physical entities. The interesting question however remains open as to whether five matrices $\gamma^{k}$ and $\gamma$ satisfying (4) and (7) are always such that whenever they decompose into a direct sum of irreducible sets, the representation $R$ can be simultaneously decomposed to correspond. We shall assume that the $\gamma$ 's occurring in (6) are in fact of this type, so that they can be considered as the direct sum of irreducible sets each independent of the other.

We can now establish

Theorem 1. A non-singular Hermitian $\nu \times \nu$-matrix $D$ always exists such that

$$
T^{\dagger} D T=D
$$

for every matrix $T$ representing a transformation of the orthochronous Lorentz group $\mathfrak{L}^{\uparrow}$.

To see this we make a transformation of the type (8) so that the representation $R$ appears in the fully reduced form (3), each representation on the right of (3) being irreducible. Now it is well known that for every irreducible representation $\Omega(n, m)$ of the othochronous Lorentz group, a non-singular Hermitian matrix $D(n, m)$ exists which has the property $T^{\dagger}(n, m)$ $\times D(n, m) T(n, m)=D(n, m)$ where $T(n, m)$ is the matrix corresponding to $T$ in this representation. ${ }^{7}$ This matrix $D(n, m)$ is uniquely defined but for multiplication by an arbitrary number. The matrix $D$ in (9) is just a direct sum of all such matrices $D(n, m)$ and is arbitrary only to the extent that each one of its sub-matrices can be multiplied by an arbitrary numerical factor $c$,

$$
D=c D(n, m)+c^{\prime} D\left(n^{\prime}, m^{\prime}\right)+\cdots .
$$

Thus, whenever the representation $R$ is brought to its fully reduced form (3), so also is $D$ brought to the corresponding form (10). The matrix $D$, being non-singular, has an inverse.

We can now define five new matrices $\alpha^{k}$ and $\beta$ through the relations

$$
\gamma^{k}=D \alpha^{k}, \quad \gamma=D \beta
$$

and obtain from (6)

Theorem 2. The most general quadratic terms in $\psi$ in the Lagrange function can be written in the form

$$
\psi+D\left(\alpha^{k} p_{k}+\beta x\right) \psi,
$$

where the five matrices $\alpha^{k}$ and $\beta$ transform according to

$$
T^{-1} \alpha^{k} T=t^{k} \alpha^{l}, \quad T^{-1} \beta T=\beta,
$$

and $D$ is a non-singular Hermitian matrix transforming according to (9).

Equation (13) follows from (4), (7), and (9). Further, the Hermiticity of $\gamma^{k}, \gamma$, and $D$ leads to the relations

$$
\alpha^{k \dagger} D=D \alpha^{k}, \beta^{\dagger} D=D \beta .
$$

The advantage of working with the $\alpha$ 's and $\beta$ instead of the $\gamma$ 's is that for any product of the $\alpha$ 's and $\beta$, say $\alpha^{k_{1}} \alpha^{k_{2}} \ldots$, we have the relation

$$
\begin{aligned}
T^{-1} \alpha^{k_{1}} \alpha^{k_{2}} \cdots T=\left(T^{-1} \alpha^{k_{1}} T\right) & \left(T^{-1} \alpha^{k_{2}} T\right) \cdots \\
=t_{l_{1}}{ }^{k_{1}} t_{2} l_{2} & \cdots \alpha^{l_{1}} \alpha^{l_{2}} \cdots,
\end{aligned}
$$

whereas no such relation holds for the $\gamma^{\prime}$ s since $T^{\dagger}$ and not $T^{-1}$ appears on the left of (4) and (7). The $\alpha$ 's and $\beta$ and their sums and products form a relativistically invariant algebra which is easier to study than the algebra generated by the $\gamma$ 's which is not relativistically invariant, although the $\gamma$ 's themselves are in the sense of Eqs. (4) and (7).

7 The existence of this matrix $D(n, m)$ corresponds to the fact that by contracting the indices of a spinor with those of the conjugate complex spinor one can form a real absolute invariant of the orthochronous Lorentz group $\mathcal{L}$. It is important to note that $D(n, m)$ can be, and has to be, so chosen that (9) holds also for the orthochronous improper transformations. 
Any representation $R$ of the orthochronous Lorentz group $\mathcal{L}^{\uparrow}$ is determined completely by its nucleus consisting of the six infinitesimal transformations $I^{k l}$ skewsymmetric in the indices $k$ and $l$, and the matrix $R_{s}$ representing the transformation which reverses the directions of the three-space axes. The six I's satisfy the well-known commutation relations

$$
\left[I^{i j}, I^{k l}\right]=-g^{i k} I^{i l}+g^{i l} I^{j k}+g^{j k} I^{i l}-g^{i l} I^{i k},
$$

where $[a, b]$ denotes $a b-b a$. Conversely, any six matrices satisfying (16) and

$$
I^{k l} R_{s}=R_{s} I^{k l}, \quad I^{0 k} R_{s}=-R_{s} I^{0 k}, \quad k, l=1,2,3,
$$

determine a representation of the orthochronous Lorentz group. The relations (17) are but a special case of

$$
T^{-1} I^{i j} T=t_{k}{ }^{i} t_{l}^{i} I^{k l} \text {, }
$$

$T$ being any matrix of the representation. As is well known, the $\alpha$ 's, $\beta$, and $I$ 's satisfy the commutation relations

$$
\begin{aligned}
{\left[\alpha^{j}, I^{k l}\right] } & =g^{j k} \alpha^{l}-g^{j l} \alpha^{k} \\
{\left[\beta, I^{k l}\right] } & =0,
\end{aligned}
$$

which follow from (13). Also,

$$
\begin{aligned}
{\left[\beta, R_{s}\right]=0, \quad\left[\alpha^{0}, R_{s}\right] } & =0, \\
& \alpha^{k} R_{s}+R_{s} \alpha^{k}=0, \quad k=1,2,3 .
\end{aligned}
$$

The general structure of the $\alpha$ 's has been given by me in an earlier paper. ${ }^{8}$ We first consider the representation $\mathbb{R}$ brought to its fully reduced form (3), and write the corresponding sub-matrices of $\alpha^{k}$ in the obvious notation $\left(n, m\left|\alpha^{k}\right| n^{\prime}, m^{\prime}\right)$. Then it has been shown in the paper quoted that every sub-matrix $\left(n, m\left|\alpha^{k}\right| n^{\prime}, m^{\prime}\right)$ must be zero unless $n^{\prime}=n \pm 1, m^{\prime}=m$, or $n^{\prime}=n, m^{\prime}=m \pm 1$, the usual condition $n^{\prime} \geqslant m^{\prime} \geqslant 0$ being always fulfilled. An exception occurs in the case $m=\frac{1}{2}$, the sub-matrix $\left(n, \frac{1}{2}\left|\alpha^{k}\right| n, \frac{1}{2}\right)$ being not necessarily zero. A consequence of (14) is that

$$
\begin{aligned}
\left(n^{\prime}, m^{\prime}\left|\alpha^{k}\right| n, m\right) & =D^{-1}\left(n^{\prime}, m^{\prime}\right)\left(n^{\prime}, m^{\prime}\left|\alpha^{k \dagger}\right| n, m\right) D(n, m) \\
& =D^{-1}\left(n^{\prime}, m^{\prime}\right)\left(n, m\left|\alpha^{k}\right| n^{\prime}, m^{\prime}\right) D(n, m)
\end{aligned}
$$

showing that $\left(n^{\prime}, m^{\prime}\left|\alpha^{k}\right| n, m\right)$ is zero if and only if $\left(n, m\left|\alpha^{k}\right| n^{\prime}, m^{\prime}\right)$ vanishes.

The matrix $\beta$ commutes with every matrix of $R$ and hence whenever $R$ is in its fully reduced form (3), $\left(n^{\prime}, m^{\prime}|\beta| n, m\right)=0$ unless $n^{\prime}=n, m^{\prime}=m$. These nonvanishing sub-matrices of $\beta$ must be arbitrary multiples of the unit matrix since the representations $R(n, m)$ are irreducible. $\beta$ can therefore always be brought to the diagonal form when $R$ is in the form (3). ${ }^{10}$ These arbi-

\footnotetext{
${ }^{8}$ H. J. Bhabha, Rev. Mod. 17, 200 (1945).

9 This relation corresponds to $(66)$ and $(67)$ of the abovementioned paper or to (30) of the present paper.

${ }_{10}$ If the same representation $\mathcal{R}(n, m)$ occurs more than once in the reduction (3), then all the non-vanishing sub-matrices of $\beta$ need not be the diagonal ones. However, even in this case it can be shown that by a suitable transformation $\beta$ can be brought to a form in which its only non-vanishing sub-matrices are on the diagonal, at the same time preserving the fully reduced form of $\mathcal{R}$ and of the corresponding form (10) of $D$.
}

trary multiplying factors can all be absorbed into $D$ since $D$ is arbitrary to this extent, so that by a suitable choice of $D$ we can always arrange that $\beta^{2}=\beta$.

Theorem 3. A transformation exists which turns $\beta$ into an idem potent matrix satisfying $\beta^{2}=\beta$. Its only eigenvalues are 0 or 1 . If it is non-singular, it is the unit matrix.

As in the case of the $\gamma$ 's, the only form of reducibility which is of interest is when the $\alpha$ 's, $I$ 's, and $R_{s}$ form a reducible set. I think it likely that such a set is always fully reducible, if it is reducible, but the statement remains to be proved. In any case, we shall asume that for the equations of interest in physics, this statement is true, so that the quadratic terms in (12) become the sum of a number of independent parts. We label quantities connected with each such part by a Greek index in brackets, thus : $\alpha_{(\mu)}, \psi_{(\mu)}$, etc.

It follows from the three preceding paragraphs that for an irreducible expression of the type (12) all the representations $R(n, m)$ occurring in $R$ have only integral values of $n, m$ or only half-odd integral values. We say the former describes a particle of integral spin, the latter a particle of half-odd-integral spin.

Even if the whole set of 11 matrices is irreducible, it does not follow that the $\alpha$ 's by themselves form an irreducible set. Indeed, examples can be given in which it is not so, though in the equations which have been studies so far the $\alpha$ 's do form an irreducible set. When the $\alpha$ 's form an irreducible set it is possible to express every matrix of the same degree as a polynomial in them, and this is true in particular for the $I$ 's and $R_{s}$. The transformation properties of products of the $\alpha$ 's as given by (15) and of the I's as given by (18) then requires that the connection should take the form ${ }^{11}$

$$
I^{k l}=\sum\left(\alpha^{\cdots} \cdots \alpha^{k} \cdots \alpha^{l} \cdots \alpha^{-}-\right),
$$

where the dots stand for an unspecified number of $\alpha$ 's, the index of each such $\alpha$ being contracted with that of another in the same term. The minus sign in the bracket indicates that a similar term with $k$ and $l$ interchanged is to be subtracted. The summation is over all terms of this type multiplied by numerical coefficients. This statement remains true when the $\alpha$ 's generate a semisimple algebra, as is the case when they are the direct sum of irreducible sets of matrices.

The variation with respect to $\psi^{\dagger}$ of the integral of (12) over a four-dimensional volume leads to the equation

$$
\left(\alpha^{k} p_{k}+\beta \chi\right) \psi=0,
$$

where the matrix $D$ has been eliminated by multiplication with $D^{-1}$ from the left. The equation for $\psi^{\dagger}$ is obtained by taking the variation with respect to $\psi$ and is the Hermitian conjugate of (22). It is also the adjoint of the equation for $\psi$.

It can be deduced from (22) and its Hermitian con-

\footnotetext{
${ }^{11}$ Harish-Chandra, Phys. Rev. 71, 793 (1947).
} 
jugate that the real four-vector

$$
s^{k}=\psi^{\dagger} D \alpha^{k} \psi
$$

satisfies the conservation equation

$$
\partial_{k} s^{k}=0
$$

and can therefore be interpreted as the charge-current vector of the system. Similarly, it can be deduced from the dependence of the variation of the Lagrangian on variations of the boundary of the region of integration that the tensor

$$
T^{k l}=\psi^{\dagger} D \alpha^{l} p^{k} \psi
$$

satisfies the conservation equation

$$
\partial_{l} T^{k l}=0 .
$$

It can therefore be interpreted as the energy-momentum tensor of the field. It has been shown by Pauli ${ }^{12,13}$ that it can be made symmetric in $k$ and $l$ by using the properties of the angular momentum tensor of the field.

\section{INTERACTION TERMS}

Next consider the cubic terms in $\psi$ in the Lagrange function. The simplest way of constructing these is to take a vector quadratic in $\psi$ of the type (23) belonging to an irreducible part of (12), say $\psi^{\dagger} \gamma_{(\mu)}{ }^{k} \psi$, where the lower index $(\mu)$ denotes the sub-matrices of $\gamma^{k}$ corresponding to the irreducible part, and contract it with one of the vectors linear in $\psi$ mentioned at the beginning of Section II which picks out components of $\psi$ belonging to another independent part of (12). Thus,

$$
e\left(\psi^{\dagger} \gamma_{(\mu)}{ }^{k} \psi\right)\left(\Gamma_{(\nu) k} \psi\right),
$$

where $e$ is an arbitrary universal constant, is a term of the third degree in $\psi$ which may be added to the Lagrange function. If it is not real, then its complex conjugate must be likewise added. The effect of a term of this sort is that the sets of components of $\psi$ namely $\psi_{(\mu)}$ and $\psi_{(\nu)}$ which were determined separately and independently of each other by two distinct sets of equations as far as the quadratic terms in (12) are concerned, now become connected and influence each other. Thus, all terms of the third or higher degree may be referred to as interaction terms. The interaction of electrons with the electromagnetic field is of this type.

If there are two independent parts in (12) with the same transformation properties, then we could manifestly combine the part $\psi_{(\nu)}$ of the wave function corresponding to one, with the part $\psi_{(\mu)}$ of the other to form a vector $\psi^{\dagger} \gamma_{(\mu \nu)}{ }^{k} \psi$ and contract this with a linear vector belonging to a third part, say, $\Gamma_{(\rho)}{ }^{k} \psi$, thus getting a term

$$
\left(\psi^{\dagger} \gamma_{(\mu \nu)}^{k} \psi\right)\left(\Gamma_{(\rho) k} \psi\right) .
$$

A term of this type involves the interaction of three

${ }^{12}$ W. Pauli, Rev. Mod. Phys. 13, 203 (1941).

${ }^{13}$ H. J. Bhabha, Proc. Ind. Acad. Sci. A21, 241 (1945). different physical entities. The usual interaction of mesons with the proton and neutron is of this type.

Interaction terms of the fourth degree between two different entities are always possible, for one can always add to the Lagrange function the invariant

$$
\left(\psi^{\dagger} \gamma_{(\mu)}^{k} \psi\right)\left(\psi^{\dagger} \gamma_{(\nu) k} \psi\right)
$$

It may be possible to introduce an interaction of this form between four different physical entities, as for example, $\left(\psi^{\dagger} \gamma_{(\mu \nu)}{ }^{k} \psi\right)\left(\psi^{\dagger} \gamma_{(\rho \sigma) k} \psi\right)$. The original interaction between electrons, neutrinos, protons, and neutrons introduced by Fermi in the theory of the $\beta$-decay was of this type.

We have so far mentioned interaction terms in which each quantity in the product is a vector, but this is clearly quite immaterial. A quadratic quantity $\psi^{\dagger} \gamma_{(\mu \nu)} \cdots \psi$ transforming according to some irreducible representation $R(n, m)$ may be contracted with a linear term $\Gamma_{(\rho)} \cdots \psi$ transforming according to the same representation to introduce a third degree interaction term in the Lagrange function. A similar remark applies to terms of the fourth or higher degree. The differential operator $p_{k}$ may also appear once in any interaction term, but our basic postulates would exclude it appearing more than once in any term, or a differential of higher order than the first occurring. This remark has a bearing on modifications of Fermi's theory of the $\beta$-decay which have been considered by several authors and would exclude most of the proposed modifications of the interaction.

It may be possible to introduce third or higher degree terms in the Lagrange function involving only one irreducible set, say $\psi_{(\mu)}$, of the components of $\psi$. For example, fourth degree terms of the type (28) with $\mu=\nu$ can invariably be introduced in the Lagrange function. Higher degree terms of this sort are of an essentially different nature from the interaction terms that have been considered so far. For in the case of the latter, the system as a whole still possesses particular solutions in which all the components of $\psi$ are zero with the exception of one irreducible set, and this set is then just a solution of the corresponding linear equation (22). The consideration of the solutions of the linear equation (22) derived only from the quadratic terms (12) in the Lagrange function is therefore of physical significance. An example is provided by the plane wave solutions of the Maxwell field which still remain exact solutions of the system as a whole, including interaction with charged particles when no such charged particles are present. On the other hand, the third or higher degree terms involving only one irreducible set of the components of $\psi$ introduce essential non-linearities in the equations, and the consideration of solutions of the linear equation (22) for such components of $\psi$ would have no physical or mathematical significance, since there are no circumstances in which the effect of the higher degree terms can be neglected. Such terms however do not seem to have played any part in physics 
so far and it may be that they do not correspond to anything in nature. The present approach to the problem therefore shows the logical significance of linear equations of the type (22), allows a distinction to be made between the usual non-linear inreraction terms and terms which would introduce essential non-linearities in the equations, and provides a justification of the usual methods of perturbation theory.

The remarks of the preceding paragraph are true classically, but have to be modified in quantum theory due to the zero point fluctuations of the field and the process of virtual pair-creation. However, the distinction between (non-linear) interaction terms and essentially non-linear terms is so deep mathematically that one would expect some essential features of this distinction to be preserved even in the quantum theory.

When we are confronted with a situation in which a number of different physical entities transform into each other, as for example, in meson decay, the question arises as to what really constitutes an elementary physical entity. The present approach allows a logical answer to be given to this question.

Definition 1. An elementary physical entiry is one whose behavior is determined by one of the independent and irreducible parts in (12), that is, in the quadratic terms in the Lagrange function. Equivalently, it is determined by an irreducible equation of the type (22).

This is the least that can be demanded of any physical entity that is to be called elementary, for if this condition is not satisfied, then the quadratic terms in the Lagrange function and the corresponding equations can be split into two independent parts and sets, respectively, which determine their parts of the wave function independently of each other. The further discussion will show that this criterion for an elementary physical entity is necessary but not sufficient.

A further sharpening of the concept of irreducibility and therefore of an elementary physical entity is possible. When the interaction terms in the Lagrange function are neglected, the different states of the same physical entity in the sense defined above cease to be connected with each other. By the state of a physical entity one means here a solution of (22). It would be absurd, however, to regard the different states as representing different physical entities simply because no transitions between them are possible in the absence of interaction. A state is not a relativistically invariant concept, and one state can be transformed into another by simply changing the frame of observation. We can therefore make the sharpened

Definition 2. An elementary physical entity is the ensemble of all solutions of Eq. (22) which can be transformed into each other by transformations of the full Lorentz group, that is, by an ensemble which is invariant and irreducible for all such transformations.

If the group mentioned in this definition were substituted by the orthochronous Lorentz group, then the positron and electron would become two distinct physi- cal entities, even though the same according to definition 1. It will, however, be shown in the next section that every equation of the type (22) which is invariant for the orthochronous Lorentz group $\mathfrak{L}^{\uparrow}$ must also be invariant for the full group so that it is legitimate to use the full Lorentz group in the definition 2 of an elementary physical entity. The electron and positron are then to be considered as different states of the same physical entity, but the states of different rest mass of a physical entity which is elementary by the definition 1 are to be considered as different physical entities by definition 2. The ensemble of states which is invariant for transformations of the full Lorentz group provides the representation space of a non-trivial representation of the inhomogeneous Lorentz group, that is, the group consisting of all Lorentz transformations and all translations of the origin of the coordinate system. ${ }^{14}$ Definition 2 is equivalent to the statement that an elementary physical entity is constituted by an ensemble of states which provides an irreducible representation of the inhomogeneous Lorentz group.

\section{PROOF OF THE EXISTENCE OF THE ANTIPARTICLE. SPIN AND STATISTICS}

It has only been postulated that the formalism shall be covariant for all transformations of the orthochronous Lorentz group $\mathcal{L}^{\uparrow}$. We now prove

Theorem 4. Every equation of the form (22) is covariant for the full Lorentz group $\mathfrak{L}$ if it is covariant for the orthochronous Lorentz group $\mathcal{L}^{\uparrow}$.

The transformation $r$ which reverses the directions of all four axes, and therefore of the time component $x^{0}$ of a time-like vector $x^{k}$, commutes with all other transformations of the group $\&$. Every transformation of $\mathscr{L}$ is obtained by multiplying the transformations of $\mathcal{L}^{\uparrow}$ by $r$, and to prove the theorem one has to prove that a $\nu \times \nu$-matrix $R$ can be found which has the properties required of the representative of $r$.

An irreducible representation $D_{i, j}$ of the orthochronous proper Lorentz group $\mathfrak{L}_{+} \uparrow$ is labeled by two positive members $i$ and $j$ each of which may take on any integral or half-odd-integral value independently of the other. The irreducible representation $R(n, m)$ of $\mathcal{L}^{\uparrow}$ decomposes if $m>0$ into two irreducible representations $D_{i, j} \dot{+} D_{j, i}$ of the proper group $\mathscr{L}_{+} \uparrow$ where $i=\frac{1}{2}(n+m), j=\frac{1}{2}(n-m)$. On the other hand, $R(n, 0)$ provides the single irreducible representation $\mathscr{D}_{n / 2, n / 2}$ of $\mathfrak{L}_{+} \uparrow$. As before, we denote the sub-matrices of $R$ with regard to this decomposition by $\left(i, j|R| i^{\prime}, j^{\prime}\right)$ and define the matrix $R$ by

$$
\left(i, j|R| i^{\prime}, j^{\prime}\right)=\left\{\begin{array}{l}
0 \text { if } i^{\prime} \neq i, j^{\prime} \neq j, \\
1(i, j) \text { if } i=i^{\prime}, j=j^{\prime} \text { and } i \\
-1(i, j) \text { if } i=i^{\prime}, j=j^{\prime} \text { and } i \text { is half } \\
\text { an odd integer }
\end{array}\right.
$$

${ }^{14} \mathrm{I}$ am indebted to Professor W. Pauli for this information, and for a very useful discussion on the subject of irreducibility in this context. 
where $1(i, j)$ denotes the unit matrix having the same number of rows and columns as the representation $D_{i, j}{ }^{15}$ Then $R^{2}=1$ and $R$ commutes with every matrix in $A$ representing a transformation of the sub-group $\mathscr{L}_{+}{ }^{\uparrow}$. Moreover, it commutes with $R_{s}$ if $n=i+j$ is an integer and anti-commutes with it if $n$ is a half-oddinteger. Since the representations with $n$ integral are single valued while those with $n$ half-odd-integral are double valued, $R$ has the correct properties for a representative of the matrix $r$. Thus, with the addition of the matrix $R$ the representation $R$ can be extended into a representation of the same degree of the full group $\&$. Moreover, with regard to the present decomposition the only non-vanishing sub-matrices of $\alpha^{k}$ and $\beta$ are ${ }^{8}$

$$
\begin{array}{lll}
\left(i, j|\beta| i^{\prime}, j^{\prime}\right) & i^{\prime}=i, & j^{\prime}=j \\
\left(i, j\left|\alpha^{k}\right| i^{\prime}, j^{\prime}\right) & i^{\prime}=i \pm \frac{1}{2}, & j^{\prime}=j \pm \frac{1}{2} .
\end{array}
$$

It follows from (29) and (30) that

$$
\alpha^{k} R=-R \alpha^{k}, \quad \beta R=R \beta,
$$

proving the theorem. ${ }^{16}$

The matrix $R_{t}=R R_{s}$ represents the transformation which reverses the direction of the time coordinate, leaving the other coordinates unchanged. Suppose (22) possesses a static solution of the type

$$
\psi=e^{i E_{0} t} \phi
$$

where the vector $\phi$ is independent of the time $t=x^{0}$. If we now reverse the direction of the time axis, Eq. (22) remains invariant as stated in the previous theorem, while the solution (32) is transformed into

$$
\psi^{\prime}=e^{i\left(-E_{0}\right) t^{\prime}} \phi^{\prime} \quad \text { where } \quad \phi^{\prime}=R_{t} \phi .
$$

This proves

Theorem 5. If Eq. (22) possesses a static positive frequency solution of type (32) then it also possesses a negative frequency solution of type (33). These negative frequency solutions must be interpreted to describe the antiparticle.

The proof just given shows that within the framework of our basic postulates every particle must have an antiparticle. So far no negative proton, for example, has been recognized in nature, but this is not a proof that it does not exist since even theoretically the chance of its occurring is small. However, if it should be established that any of the elementary particles, say the proton, has no antiparticle in nature, then our proof shows that the basic equation could not be of the type (22), and it would be necessary to abandon at least one of the basic postulates on which present quantum theory is built.

\footnotetext{
15 That the only non-vanishing sub-matrices $(i, j|R| i, j)$ must be multiples of the unit matrix follows from the fact that $R$ must commute with every $I^{k l}$ whether the representation $D_{i, j}$ is singlevalued or double-valued.

${ }_{16}$ The form (29) is uniquely determined by the first relation of (31) but for a multiplying factor if the $\alpha$-matrices by themselves form an irreducible set.
}

It remains to consider what happens when $R$ is substituted in place of $T$ in (9). A spinor symmetric in $i$ undotted and $j$ dotted indices transforms according to the irreducible representation $\mathfrak{D}_{i, j}$ of $\mathfrak{L}_{+} \uparrow$. Its complex conjugate transforms like a spinor with $i$ dotted and $j$ undotted indices, that is, according to $\mathscr{D}_{j, i}$. Hence, with respect to the present decomposition the only nonvanishing sub-matrices of $D$ are $(i, j|D| j, i)$. It follows from (29) that

$$
R^{\dagger} D R=\left\{\begin{array}{l}
+R \text { for } i+j=n \text { an integer } \\
-R \text { for } i+j=n \text { a half-odd-integer. }
\end{array}\right.
$$

Hence, in a representation of $\mathfrak{L}$ every matrix $T$ representing a transformation of the orthochronous group $\mathscr{L}^{\uparrow}$ satisfies (9), while every matrix representing an antichronous transformation of $\&$ satisfies (9) with a minus sign on the right-hand side. It follows that in corresponding states $\psi$ and $\psi^{\prime}$ of positive and negative frequency the charge density $\psi^{\dagger} D \alpha^{0} \psi$ at any point has the same sign if the spin is a half-odd-integer and the opposite sign if it is an integer. Correspondingly, the energy density $\psi^{\dagger} D \alpha^{0}[-i(\partial / \partial t)] \psi$ given by $(25)$ has the opposite sign for half-odd-integral and the same sign for integral spin.

This leads to

Theorem 6. In corresponding states of positive and negative frequency the total charge has the same and the total energy the opposite sign if the spin is half-odd-integral, while the total charge has the opposite and the total energy the same sign if the spin is integral.

From this theorem one can deduce the important corollary:

Neither the energy density nor the total energy can be positive definite for particles of half-odd-integral spin and neither the charge density nor the total charge can be positive definite for particles of integral spin.

The appearance of states of negative total charge or negative total energy requires one to treat the wave function $\psi$ and its Hermitian conjugate as operators and not as ordinary functions of the coordinates. Since every wave function can be expressed as a sum of all its static solutions of the type (32) and (33) multiplied by suitable coefficients, this requires that these coefficients or amplitudes should themselves be treated as operators. If it is required that the number of particles of the given type in each such state be a positive integer or zero, and that the probability of any one of these alternatives occurring is positive, the sum of all these probabilities being 1 , as required by the usual concept of probability, then it can be proved that one has to work with a positive definite metric in this amplitude space. The difficulty regarding the states of negative energy of particles of half-odd-integral spin can then be removed only by the use of Dirac's wellknown hole theory requiring the particles to obey the exclusion principle, provided the total charge is positive for all positive frequency states. On the other hand, 
Pauli $^{17}$ has shown that for particles of integral spin quantization in accordance with the exclusion principle leads to a mathematical inconsistency. This leads to the well-known conclusion: A physically sensible theory capable of a probability interpretation is possible for particles of hald-odd-integral spin only if the particles satisfy Fermi-Dirac statistics, and for particles of integral spin if they satisfy Einstein-Bose statistics.

The theory therefore requires a connection between the spin of an elementary particle and its statistics which appears to to be in agreement with nature. However, a somewhat unsatisfactory feature of this demonstration, in my opinion, is the circumstance that the connection between particles of half-odd-integral spin and Fermi-Dirac statistics is due to the necessity of a physically sensible interpretation-mathematically the theory could be quantized according to BoseEinstein statistics-while the connection between particles of integral spin and Bose-Einstein statistics is due to an entirely different type of reason, namely, the mathematical impossibility of quantizing the theory according to FermiDirac statistics.

The theoretical connection between spin and statistics disappears altogether if one does not work with finite representations, that is, with a wave function $\psi$ in (1) having only a finite number of components. As is well known ${ }^{18,19}$ there exist "infinite" representations of the Lorentz group, that is, representations by matrices having a discretely infinite number of rows and columns. These representations are all unitary, whereas the finite representations can never be unitary. It has been shown by Dirac ${ }^{18}$ and Harish-Chandra ${ }^{19}$ that these infinite representations can also be made the basis of wave equations of the type (22). Due to the unitary character of the infinite representations, particles of integral and halfodd-integral spin can then be quantized according to BoseEinstein or Fermi-Dirac statistics at will.

When one comes to review the experimental position, one finds that definite evidence is extraordinarily meager. One knows definitely that the electron, proton, and neutron all have half-odd-integral spins and satisfy the Fermi-Dirac statistics. The present evidence, derived from non-relativistic experiments as far as concerns the proton and neutron, is that each of these particles has a spin $\frac{1}{2} \hbar$. The experimental evidence then simply reduces to the more limited statement that every elementary particle of spin $\frac{1}{2} \hbar$ satisfies FermiDirac statistics. Up to now there is no positive evidence that elementary particles of half-odd integral spin greater than $\frac{1}{2} \hbar$ exist in nature, though in my opinion the possibility remains that the proton, for example, may possess unstable states of higher spin and higher mass into which it might be thrown after a violent collision with another particle while moving with an extremely relativistic velocity.

The evidence with regard to particles of integral spin is even more meager. One knows definitely that the photon has an integral spin $\hbar$ and obeys Bose-Einstein statistics. The photon, however, has no rest mass. There is so far no definite evidence that an elementary particle of finite rest mass and integral spin exists in

${ }^{17}$ W. Pauli, Phys. Rev. 58, 716 (1940).

18 P. A. M. Dirac, Proc. Roy. Soc. A183, 284 (1945).

${ }_{19}$ Harish-Chandra, Proc. Roy. Soc. A189, 372 (1947). nature, although there are theoretical reasons for believing that some of the mesons might be of this type. About the statistics of the mesons absolutely nothing is known experimentally and only theoretical inferences can be made.

\section{GENERAL COMMUTATION RULES OF THE $\alpha$-MATRICES. REST MASS}

Introduce the abbreviations

$$
P=\alpha^{k} p_{k}, \quad p^{2}=g^{k l} p_{k} p_{l}=p_{k} p^{k} .
$$

In this section the $p_{k}$ stand for any four real numbers. Put

$$
\psi=\exp \left(i p_{k} x^{k}\right) \phi
$$

where $\phi$ is an one-column matrix whose components do not depend on the coordinates.

$P$ being a matrix of finite degree, satisfies a minimal equation $^{20}$ of finite degree whose coefficients must be polynomials of the four numbers $p_{k}$ since $P$ itself is a linear function of $p_{k}$. Moreover, (13) shows that a Lorentz transformation transforms $P$ into

$$
P^{\prime}=\alpha^{k} p_{k}{ }^{\prime}=T P T^{-1},
$$

so that $P^{\prime}$ satisfies the same minimal equation as $P$. The minimal equation is therefore invariant for all Lorentz transformations, and its coefficients must therefore contain $p_{k}$ in an invariant combination. The only such invariant which can be formed from $p_{k}$ is $p^{2}$ and functions of it. Since the equation must be homogeneous in the $p_{k}$ and the invariant of lowest degree is the quadratic expression $p^{2}$, it follows that the equation must contain only even, or only odd powers of $P$. Every such equation can be factorized into linear factors of $P^{2}$ (in the field of all complex numbers). It can therefore always be written in the form

$$
\left(P^{2}-a_{1}^{2} p^{2}\right)\left(P^{2}-a_{2}^{2} p^{2}\right) \cdots=0
$$

if its degree $d$ is even, or $P$ times (38) if $d$ is odd, the $a$ 's being certain numbers independent of $p_{k}$. Equation (38) can be written in the form

$$
p_{i} p_{j} p_{k} p_{l} \cdots\left(\alpha^{i} \alpha^{j}-a_{1}^{2} g^{i j}\right)\left(\alpha^{k} \alpha^{l}-a_{2}^{2} g^{k l}\right) \cdots=0 .
$$

Since this is a polynomial of degree $d$ in the four numbers $p_{0}, p_{1}, p_{2}$, and $p_{3}$ which holds for arbitrary values of these numbers, the coefficient of every term of it must vanish. This yields

Theorem 7. Every set of $\alpha$-matrices must satisfy an algebraic equation of degree $d$ of the type ${ }^{11}$

$$
\sum\left(\alpha^{i} \alpha^{j}-a_{1}^{2} g^{i j}\right)\left(\alpha^{k} \alpha^{l}-a_{2}^{2} g^{k l}\right) \cdots=0,
$$

if $d$ is even, or $\alpha^{h}$ times (40) if $d$ is odd, where $\sum$ denotes a summation over similar terms with all possible permutations of the indices $i, j, k, l, \cdots$ and $a_{1}{ }^{2}, a_{2}{ }^{2}, \cdots$ are certain numbers. $d$ is the degree of the minimal equation of any matrix of the set.

${ }^{20}$ The minimal equation of a matrix is the algebraic equation of least degree satisfied by that matrix. 
In particular, the minimal equation of $\alpha^{k}$ is obtained by putting all the indices equal to $k$ in (40), and is

$$
\left\{\left(\alpha^{k}\right)^{2}-a_{1}^{2} g^{k k}\right\}\left\{\left(\alpha^{k}\right)^{2} a_{2}^{2} g^{k k}\right\} \cdots=0,
$$

if $d$ is even, or $\alpha^{k}$ times (41) if $d$ is odd.

Consider first those equations of the form (22) in which $\beta$ is non-singular, so that it can be replaced by the unit matrix according to theorem 3 . Then for every solution $\psi$ of (22),

$$
(P+\chi) \psi=0 \text {, whence } P^{n} \psi=(-\chi)^{n} \psi .
$$

Operating on a solution $\psi$ with the left-hand side of (38) replaces every $P$ in it by $-\chi$. Since the resulting operator is then a multiple of the unit matrix, it must itself vanish by (38). Hence, ${ }^{21}$

$$
\left(\chi^{2}-a_{1}^{2} p^{2}\right)\left(\chi^{2}-a_{2}^{2} p^{2}\right) \cdots=0
$$

if $d$ is even, or $\chi$-times (43) if $d$ is odd, proving

Theorem 8. Equation (32) (with $\beta=1$ ) has plane wave solutions of the type (36) only if $p^{2}$ has one of the values $\chi^{2} / a_{1}{ }^{2}, \chi^{2} / a_{2}{ }^{2}, \cdots$ where $\pm a_{1}, \pm a_{2}, \cdots$ are the non-zero eigenvalues of $\alpha^{0}$. Conversely, for every such eigenvalue of $\alpha^{0}$ a solution of the type (36) exists.

To prove the converse, put $p^{2}=\chi^{2} / a_{r}{ }^{2}$ in (38). Then (38) contains a factor $P^{2}-\chi^{2}$ and it can be written

$$
(P+\chi)\left\{(P-\chi)\left(P^{2}-\frac{a_{1}^{2}}{a_{r}^{2}} \chi^{2}\right)\left(P^{2}-\frac{a_{2}^{2}}{a_{r}^{2}}-\chi^{2}\right) \cdots\right\}=0 .
$$

Any column of the matrix in curly brackets ${ }^{22}$ yields the required solution of (32) for which $p^{2}=\chi^{2} / a_{r}^{2}$.

The rest mass of a particle is defined as the positive root of the eigenvalues of the operator $p^{2}$. One can then draw the following conclusions from theorem 8 .

Lemma 9. If a particle is to have only real values of the mass, then all the eigenvalues $\pm a_{1}, \pm a_{2}, \cdots$ of $\alpha^{0}$ must be real.

Lemma" 10. If the particle is to have only one real mass, then $\alpha^{0}$ must have one and only one pair of non-zero real eigenvalues $\pm a_{1}$ (which may occur several times).

Without loss of generality one may put $a_{1}=1$ since this can be achieved by changing the value of $\chi$ and a renormalization of $\psi$. The minimal equation of $\alpha^{0}$ is then

$$
\left\{\left(\alpha^{0}\right)^{2}-1\right\}^{r}\left(\alpha^{0}\right)^{s}=0,
$$

where $r \geqslant 1, s \geqslant 0$ are non-negative integers.

Lemma 11. If the particle is to have zero rest mass, then $\beta$ in (22) must be singular.

It may, but need not, be zero. Hence, all equations for particles of zero rest mass are not obtained by putting $x=0$ in the corresponding equation for a particle of finite mass, and irreducible equations exist in which $\beta$ is singular but not zero. It may be found later

\footnotetext{
${ }^{21}$ H. J. Bhabha, Curr. Sci. India. 14, 89 (1945).

${ }^{22}$ This matrix cannot be identically zero for it would mean that $P$ satisfied a minimal equation of degree $d-1$, contrary to as-
} sumption. that in nature particles of spin greater than $\frac{1}{2}$ can exist if the rest mass is zero, but not if it is finite.

Equation (38) being an identity in the $p_{k}$, holds even when we put $p_{k}=-i \partial_{k}$. Equation (43) is then to be regarded as an operator equation satisfied by every component of the wave function $\psi$. Since $p^{2}=-\partial_{k} \partial^{k}$, it follows that every component of $\psi$ satisfies a differential equation of order higher than the second unless $r=1$.

\section{THE EQUATION OF DIRAC}

The work of the last section allows one to tabulate the various relativistic wave equations according to the degree of the minimal equation of their $\alpha$-matrices. For brevity we call the degree $d$ of the minimal equation of the $\alpha$-matrices of an equation of type (22) the "algebraic degree" of the wave equation. One can regard an equation as simpler than another if its algebraic degree is less.

A minimal equation of degree 1 of the type (41) leads to the trivial solution $\alpha^{0}=\alpha^{1}=\alpha^{2}=\alpha^{3}=0$, and in consequence no wave equation of the type (22) is possible.

The next simplest case is an equation of degree 2 . Since $\alpha^{k}$ and its Hermitian conjugate $\alpha^{k \dagger}$ are similar by (14), if $a_{1}$ is an eigenvalue, then $\bar{a}_{1}$ must be one also. Hence, when the degree $d$ is less than four, the only two possibilities are in any case $a_{1}$ purely real or purely imaginary. Without loss of generality one can therefore put $a_{1}=1$ for it will be shown below that the only other alternative $a_{1}=i$ does not satisfy postulate $1^{\prime}$. Equation (40) then reduces to

$$
\alpha^{i} \alpha^{j}+\alpha^{j} \alpha^{i}=2 g^{i j} .
$$

These are just the commutation relations of the Dirac matrices. As is well known, a set of quantities satisfying (45) has only one irreducible representation, namely, by matrices with four rows and columns. By a use of (45) every relation of the type (21) can be reduced to

$$
I^{k l}=c\left(\alpha^{k} \alpha^{l}-\alpha^{l} \alpha^{k}\right),
$$

$c$ being some number, and this is the simplest possible connection between the $\alpha$ 's and I's. Moreover, the representation of the Lorentz group provided by the transformations of the wave function $\psi$ is then the basic spinor representation and is irreducible. It follows from (13) that the matrix $\beta$ in (22) must necessarily be a multiple of the unit matrix. There is therefore one and only one equation of type (22) for which the $\alpha$ 's satisfy a minimal equation of degree 2, namely, the famous equation of Dirac. Our derivation shows that it has a unique position in the scheme of relativistic wave equations, being the simplest equation which is possible within the framework of our basic postulates. As is well known it describes a particle of spin $\frac{1}{2}$.

Since $\beta$ has to be a multiple of the unit matrix in this case, it must be zero if it is singular. We conclude: There is only one possible equation for a particle of $\operatorname{spin} \frac{1}{2}$ 
and zero rest mass, namely (22) with $\chi=0$ and the $\alpha$ 's satisfying (45).

If $a_{1}=i$ the sign on the right of (45) is reversed, and then the one and only representation of the $\alpha^{\prime}$ s can be so chosen that $\alpha^{0}$ is skew-Hermitian while $\alpha^{1}, \alpha^{2}$, and $\alpha^{3}$ are Hermitian. It then follows from (14) that $D$ must anticommute with $\alpha^{0}$, and commute with $\alpha^{1}, \alpha^{2}$, and $\alpha^{3}$, whence $D=i \alpha^{1} \alpha^{2} \alpha^{3}$. But the transformation $r_{s}$ which reverses the direction of the threespace axes is represented by a multiple $c$ of $\alpha^{0}$, from which follows $R_{s}^{\dagger} D R_{s}=-\bar{c} \alpha^{0}\left(i \alpha^{1} \alpha^{2} \alpha^{3}\right) c \alpha^{0}=-\bar{c} c D$ proving that (9) can never be satisfied. The Lagrangian would therefore not be invariant for all orthochronous transformations, contrary to postulate $1^{\prime}$.

VII. EQUATIONS FOR PARTICLES OF SPIN 0 AND 1. A NEW EQUATION

The next simplest equations are provided by a set of $\alpha$ 's satisfying an equation of the type (40) of degree 3, namely,

$$
\begin{array}{r}
\alpha^{i} \alpha^{j} \alpha^{k}+\alpha^{i} \alpha^{k} \alpha^{i}+\alpha^{j} \alpha^{i} \alpha^{k}+\alpha^{k} \alpha^{i} \alpha^{j}+\alpha^{j} \alpha^{k} \alpha^{i}+\alpha^{k} \alpha^{j} \alpha^{i} \\
=2 g^{i j} \alpha^{k}+2 g^{j k} \alpha^{i}+2 g^{k i} \alpha^{j} .
\end{array}
$$

This relation is more general than the Duffin commutation relation

$$
\alpha^{i} \alpha^{j} \alpha^{k}+\alpha^{k} \alpha^{j} \alpha^{i}=g^{i j} \alpha^{k}+g^{k j} \alpha^{i},
$$

although matrices satisfying (48) clearly satisfy (47) -also. It can be deduced from (48) that

$$
\left[\alpha^{i},\left[\alpha^{j}, \alpha^{k}\right]\right]=g^{i j} \alpha^{k}-g^{i k} \alpha^{j} .
$$

Further, it follows from (48) that the six quantities $I^{k l}$ defined by (46) with $c=1$ satisfy (16) and (19a). These $I$ 's can therefore be identified with the infinitesimal transformations of the corresponding representation of the Lorentz group.

Conversely, if the $\alpha$ 's satisfy (46) then together with (19) and (47) we can deduce that they must satisfy (48).

It has been shown by $\mathrm{Kemmer}^{23}$ that the algebra defined by (48) has only two non-trivial inequivalent irreducible representations. The one consisting of matrices of five rows and columns leads to the so called Klein-Gordon equation describing a particle of spin zero. The other of ten rows and columns leads to the so called Proca equation describing a particle of spin 1.

The circumstance that neither (48) nor (46) can be deduced from (47) indicates that there must be irreducible representations of the $\alpha$ 's satisfying (47) but not (48) and (46). In (47) any particular matrix, say $\alpha^{0}$, occurs on the extreme left or extreme right in more than one term, in contrast with (48). In consequence (48) by itself does not enable one to move any particular $\alpha$ by two or more places to the left or right in a product of $\alpha$ 's. The abstract algebra generated by algebraic quantities satisfying only (47) is therefore not finite and one would expect there to be an infinite

\footnotetext{
${ }^{23}$ N. Kemmer, Proc. Roy. Soc. A173, 91 (1939).
}

number of inequivalent irreducible sets of matrices satisfying (47), all except two of which will not satisfy (48). Each such set must yield an algebraically irreducible wave equation for an elementary particle of spin 1 according to definition 1 (but not definition 2). Every such particle possesses only one value of the rest mass.

One example of such equations is the following. Let $F^{k l}$ be a skew-symmetric tensor. Define a vector $A^{k}$ and a skew-symmetric tensor $A^{k l m}$ by

$$
\chi A^{k}=-\partial_{l} F^{k l}, \quad \chi A^{k l m}=\partial^{k} F^{l m}+\partial^{l} F^{m k}+\partial^{m} F^{k l},
$$

where $\chi$ is an arbitrary real number. Then the set of equations defined by (50a) and

$$
\left(\partial^{k} A^{l}-\partial^{l} A^{k}\right)+\partial_{m} A^{k l m}+\chi F^{k l}=0
$$

describes a particle of rest mass $\chi$. The fourteen quantities $A^{k}, F^{k l}, A^{k l m}$ all satisfy the equation $\left(\partial_{k} \partial^{k}\right.$ $\left.+\chi^{2}\right) \psi=0$. Writing Eqs. (50) in the form (22) with the help of four $14 \times 14$ matrices $\alpha^{k}$, it is easy to verify that the $\alpha$ 's satisfy (47). For example, $\left(\alpha^{k}\right)^{3}=g^{k k} \alpha^{k}$ and $\left(\alpha^{0}\right)^{2} \alpha^{1}+\alpha^{0} \alpha^{1} \alpha^{0}+\alpha^{1}\left(\alpha^{0}\right)^{2}=\alpha^{1}$. But $\alpha^{0} \alpha^{1} \alpha^{0} \neq 0$, proving that the matrices do not satisfy (48). Hence, the $\alpha$ 's cannot be expressed as a direct sum of $\alpha$ 's satisfying the Duffin commutation relations. The connection between the $I$ 's and the $\alpha$ 's cannot be (36) as in the cases treated by Kemmer, even though we are concerned here with a particle of maximum spin 1 . The total energy is also not positive definite.

As one goes to equations of higher algebraic degree, corresponding to particles of higher spin, the number and variety of the possible equations increases rapidly. It is, therefore, necessary to add further restricting conditions to limit the number of allowed equations. The example given above shows that if the equation of algebraic degree 1 are to be restricted to the KleinGordon and Proca equations, then one can achieve this by imposing as further condition either (a) that the connection (46) between the $I$ 's and $\alpha$ 's shall always hold, or (b) that either the total charge or the total energy shall always be positive. The scheme that arises on the basis of the restriction (a) has been fully investigated and leads to particles having several different values of the rest mass. Except for the three equations for particles of spin $0, \frac{1}{2}$, and 1 it does not include the equations for particles of higher spin given by Dirac-Fierz-Pauli. The requirement (b) includes all the equations of the Dirac, Fierz, and Pauli scheme and many others besides, and the particles are not elementary in the sense of definition 2 . This raises the interesting question as to whether the scheme of equations can be limited to include only the equations of Dirac, Fierz and Pauli by imposing suitable algebraic conditions on the $\alpha$-matrices. 